\title{
MAXIMAL FUNCTIONS ASSOCIATED WITH CURVES AND THE CALDERÓN-ZYGMUND METHOD OF ROTATIONS
}

\author{
BY
}

\author{
SHUICHI SATO
}

\begin{abstract}
Let $\delta_{t}(t>0)$ be a dilation in $\mathbf{R}^{n}(n \geqslant 2)$ defined by

$$
\delta_{t} x=\left(t^{\alpha_{1}} x_{1}, t^{\alpha_{2}} x_{2}, \ldots, t^{\alpha_{n}} x_{n}\right) \quad\left(x=\left(x_{1}, x_{2}, \ldots, x_{n}\right)\right),
$$

where $\alpha_{i}>0(i=1,2, \ldots, n)$ and $\alpha_{i} \neq \alpha_{j}$ if $i \neq j$. For $\nu \in \mathbf{R}^{n}$ with $|\nu|=1$, let $\Gamma_{\nu}$ : $(0, \infty) \rightarrow \mathbf{R}^{n}$ be a curve defined by $\Gamma_{\nu}(t)=\delta_{t} \nu(0<t<\infty)$. Using maximal functions associated with the curves $\Gamma_{\nu}$, we define an operator $M$ which is a nonisotropic analogue of the one studied in R. Fefferman [2]. It is proved that $M$ is a bounded operator on $L^{p}\left(\mathbf{R}^{n}\right)$ for some $p$ with $1<p<2$. As its application we prove the $L^{p}$ boundedness of operators of the form $T^{*}(f)(x)=\sup _{\varepsilon}>0\left|T_{\varepsilon}(f)(x)\right|$, where $T_{\varepsilon}$ is an integral operator associated with a variable kernel with mixed homogeneity.
\end{abstract}

1. Introduction. Let $\alpha=\left(\alpha_{1}, \alpha_{2}, \ldots, \alpha_{n}\right)$ be an $n$-tuple of positive numbers. We define dilations $\delta_{t}(t>0)$ by

$$
\delta_{t} x=\delta_{t}^{\alpha} x=\left(t^{\alpha_{1}} x_{1}, t^{\alpha_{2}} x_{2}, \ldots, t^{\alpha_{n}} x_{n}\right),
$$

where $x=\left(x_{1}, x_{2}, \ldots, x_{n}\right) \in \mathbf{R}^{n}(n \geqslant 2)$.

Set $S^{n-1}=\left\{x \in \mathbf{R}^{n}:|x|=1\right\}$, where $|x|$ is the Euclidean norm of $x$. For a locally integrable function $f$ defined on $\mathbf{R}^{n}$ and $\nu \in S^{n-1}$, we define maximal functions by

$$
M_{\nu}(f)(x)=M_{\nu}^{\alpha}(f)(x)=\sup _{\varepsilon>0} \frac{1}{\varepsilon} \int_{0}^{\varepsilon}\left|f\left(x-\delta_{t}^{\alpha} \nu\right)\right| d t .
$$

We set

$$
M(f)(x)=M^{\alpha}(f)(x)=\left(\int_{S^{n-1}}\left|M_{\nu}^{\alpha}(f)(x)\right|^{2} d \nu\right)^{1 / 2},
$$

where $d \nu$ is the element of area of $S^{n-1}$.

We want to know when $M^{\alpha}$ is bounded on $L^{p}\left(\mathbf{R}^{n}\right)$. R. Fefferman [2] proved the following theorem.

TheOREM A. Let $\alpha_{0}=(1,1, \ldots, 1)$. Then

$$
\left\|M^{\alpha_{0}}(f)\right\|_{p} \leqslant c_{p, n}\|f\|_{p} \quad \text { for } p>2 n /(n+1),
$$

where $\|\cdot\|_{p}$ denotes the norm on the Lebesgue space $L^{p}\left(\mathbf{R}^{n}\right)$.

If $\alpha_{1}=\alpha_{2}=\cdots=\alpha_{n}$, then we have $M^{\alpha}(f)(x) \leqslant c M^{\alpha_{0}}(f)(x)$. Thus in this case the $L^{p}$ boundedness of $M^{\alpha}$ follows from Theorem A.

Received by the editors April 2, 1985.

1980 Mathematics Subject Classification. Primary 42B25. 
From now on we suppose that $\alpha=\left(\alpha_{1}, \alpha_{2}, \ldots, \alpha_{n}\right)$ satisfies that $\alpha_{i} \neq \alpha_{j}$ if $i \neq j$. Set

$$
\tau=\min \left\{\alpha_{1}, \alpha_{2}, \ldots, \alpha_{n}\right\}, \quad \gamma=\alpha_{1}+\alpha_{2}+\cdots+\alpha_{n} .
$$

In this note we will prove the following result.

THEOREM. If

$$
p>\frac{2\{n \gamma-(n-2) \tau\}}{n \gamma-(n-4) \tau}
$$

then

$$
\left\|r M^{\alpha}(f)\right\|_{p} \leqslant c_{p, \alpha}\|f\|_{p}
$$

The proof of the theorem follows the same lines as Fefferman's proof of Theorem A and depends on the ideas of Stein and Wainger [5].

As an application of the theorem, we will prove the $L^{p}$ boundedness of certain maximal operators by the method of rotations of Calderon-Zygmund.

2. Preliminaries. For $x, y \in \mathbf{R}^{n},(x, y)$ is the ordinary inner product in $\mathbf{R}^{n}$. Let $|x|=(x, x)^{1 / 2}\left(x \in \mathbf{R}^{n}\right)$.

For a function $f$ defined on $\mathbf{R}^{n}$, set

$$
f_{t}(x)=t^{-\gamma} f\left(\delta_{t^{-1}} x\right) \quad(t>0) .
$$

For $x \in \mathbf{R}^{n} \backslash\{0\}, \rho(x)$ is the unique $t$ such that $\left|\delta_{t^{-1}} x\right|=1$, and let $\rho(0)=0$. Then $\rho(x)$ has the following properties:

$$
\begin{gathered}
\rho(x) \geqslant 0, \quad \rho(x)=0 \quad \text { if and only if } \quad x=0 ; \\
\rho\left(\delta_{t} x\right)=t \rho(x) \quad(t>0) ; \\
\rho(x)=1 \quad \text { if and only if }|x|=1 .
\end{gathered}
$$

We need the following elementary result.

LeMMA 1. Let $\Phi$ be a function defined on $\mathbf{R}^{n}$ such that

$$
|\Phi(x)| \leqslant\{1+\rho(x)\}^{-\gamma-1} \quad\left(x \in \mathbf{R}^{n}\right) .
$$

Then

$$
\left\|\sup _{t>0}\left|\Phi_{t} * f\right|\right\|_{p} \leqslant c_{p}\|f\|_{p} \quad \text { for } p>1 \text {. }
$$

This follows from a result of [3] (see [5, p. 1265]).

Let $f \in L^{1}\left(\mathbf{R}^{n}\right)$. The Fourier transform of $f$ is defined by

$$
\hat{f}(\xi)=\int_{\mathbf{R}^{n}} f(x) e^{-2 \pi i(x, \xi)} d x .
$$

The inverse Fourier transform of $f$ is defined by $\breve{f}(\xi)=\hat{f}(-\xi)\left(\xi \in \mathbf{R}^{n}\right)$.

$C_{0}^{\infty}\left(\mathbf{R}^{n}\right)$ denotes the class of infinitely differentiable functions on $\mathbf{R}^{n}$ with compact support.

Finally in this note we often use the same notation for constants which are different in different occurrences. 
3. Main Lemma. In this section, we prove the Main Lemma below, which is crucial in the proof of the theorem.

Let $\eta, \phi \in C_{0}^{\infty}\left(\mathbf{R}^{n}\right)$ be such that

$$
\begin{gathered}
\eta(\xi)=1 \quad \text { if } \rho(\xi) \leqslant 1, \quad \quad \operatorname{supp}(\eta) \subset\left\{\xi \in \mathbf{R}^{n}: \rho(\xi) \leqslant 2\right\} ; \\
\hat{\phi}(0)=1 .
\end{gathered}
$$

For a complex number $z$ and $f \in C_{0}^{\infty}\left(\mathbf{R}^{n}\right)$, set

$$
g_{z}(f)(x)=\left(\int_{0}^{\infty} \int_{S^{n-1}}\left|N_{\nu, t}^{(z)} f(x)-\phi_{t} * f(x)\right|^{2} d \nu \frac{d t}{t}\right)^{1 / 2},
$$

where

$$
\begin{gathered}
\left(N_{\nu, t}^{(z)} f\right)^{\wedge}(\xi)=\eta\left(\delta_{t} \xi\right) n_{\nu}\left(\delta_{t} \xi\right) \hat{f}(\xi)+\rho^{z}\left(\delta_{t} \xi\right)\left(1-\eta\left(\delta_{t} \xi\right)\right) n_{\nu}\left(\delta_{t} \xi\right) \hat{f}(\xi) \\
n_{\nu}(\xi)=\int_{1}^{2} e^{-2 \pi i\left(\delta_{t}, \xi \xi\right)} d t
\end{gathered}
$$

Now we are ready to state the Main Lemma.

MAIN Lemma. Let $\sigma_{1}=\tau / n$. If $\sigma_{1} / 2<\operatorname{Re} z<\sigma_{1}$, we have

$$
\left\|g_{z}(f)\right\|_{2} \leqslant c_{z}\|f\|_{2} \text {. }
$$

For $x=\left(x_{1}, x_{2}, \ldots, x_{n}\right) \in \mathbf{R}^{n}$, set

$$
m(x)=\min \left\{\left|x_{1}\right|,\left|x_{2}\right|, \ldots,\left|x_{n}\right|\right\} .
$$

The proof of the Main Lemma is based on the following estimate.

LEMMA 2. If $\nu \in S^{n-1}$, then

$$
\left|n_{\nu}(\xi)\right| \leqslant c_{\alpha}\left\{1+m(\nu) \rho^{\tau}(\xi)\right\}^{-1 / n} \quad \text { for all } \xi \in \mathbf{R}^{n},
$$

where $c_{\alpha}$ is a constant independent of $\xi$ and $\nu$.

In order to prove Lemma 2, we need the following three lemmas.

LeMma 3. Let $f_{\xi, \nu}(t)=\left(\delta_{t} \nu, \xi\right)$ for $\xi, \nu \in S^{n-1}, t>0$. Then

$$
\inf \left\{\sum_{i=1}^{n}\left|\left(\frac{d}{d t}\right)^{i} f_{\xi, \nu}(t)\right|: 1 \leqslant t \leqslant 2, \xi \in S^{n-1}\right\} \geqslant c m(\nu),
$$

where $c$ is a positive constant independent of $\nu$.

LEMMA 4. Let $f_{\xi, \nu}$ be the same function as that in Lemma 3. There exists a positive integer $K$ depending only on $n$ such that for each $f_{\xi, \nu}\left(\xi, \nu \in S^{n-1}\right)$ there is $K$ points $1<\beta_{1}<\beta_{2}<\cdots<\beta_{K}<2$ satisfying the following conditions:

(1) for each integer $i, 1 \leqslant i \leqslant K+1$, there is an integer $k, 1 \leqslant k \leqslant n$, such that

$$
\left|f_{\xi, \nu}^{(k)}(t)\right|=\sup _{1 \leqslant j \leqslant n}\left|f_{\xi, \nu}^{(j)}(t)\right| \text { for all } t \in\left(\beta_{i-1}, \beta_{i}\right)
$$

$\left(\beta_{0}=1, \beta_{K+1}=2\right)$

(2) $f_{\xi, \nu}^{\prime}(t)$ is monotone in each of the intervals $\left(1, \beta_{1}\right),\left(\beta_{1}, \beta_{2}\right), \ldots,\left(\beta_{K}, 2\right)$. 
LEMMA 5 (VAN DER CORPUT). Let $f$ be a real-valued function on the interval $[a, b]$. Suppose that $f$ is $j$ times continuously differentiable $(j \geqslant 1)$ on $[a, b]$.

(1) If $f^{\prime}(t)$ is monotone, and if there is a positive $\lambda$ such that $\left|f^{\prime}(t)\right| \geqslant \lambda$ for all $t \in[a, b]$, then

$$
\left|\int_{a}^{b} e^{2 \pi i f(t)} d t\right|<\lambda^{-1} .
$$

(2) If $j \geqslant 2$, and if there is a positive $\rho$ such that $\left|f^{(j)}(t)\right| \geqslant \rho$ for all $t \in[a, b]$, then

$$
\left|\int_{a}^{b} e^{2 \pi i f(t)} d t\right| \leqslant c_{j} \rho^{-1 / j}
$$

Lemma 4 is an immediate consequence of Stein-Wainger [4, Lemma 3]. We omit the proof.

Lemma 5 is given in Stein-Wainger [5, p. 1258].

Proof of Lemma 3. We may assume that $m(\nu)>0$. Set

$$
a_{i j}(t, \nu)=\nu_{j}(d / d t)^{i} t^{\alpha_{1}}
$$

for $1 \leqslant i \leqslant n, 1 \leqslant j \leqslant n$. Let $A(t, \nu)$ be the $n \times n$ matrix whose $(i, j)$-component is given by $a_{i j}(t, \nu)(1 \leqslant i, j \leqslant n): A(t, \nu)=\left(a_{i j}(t, \nu)\right)$.

For $x=\left(x_{1}, x_{2}, \ldots, x_{n}\right) \in \mathbf{R}^{n}$, define $A(t, \nu) x \in \mathbf{R}^{n}$ by

$$
A(t, \nu) x=\left(\sum_{j=1}^{n} a_{1 j}(t, \nu) x_{j}, \sum_{j=1}^{n} a_{2 j}(t, \nu) x_{j}, \ldots, \sum_{j=1}^{n} a_{n j}(t, \nu) x_{j}\right) .
$$

Then, since det $A(t, v) \neq 0$ (see (3.4) below), we have

$$
\inf _{\xi \in S^{n-1}}|A(t, \nu) \xi| \leqslant c_{1}\left\|A(t, \nu)^{-1}\right\|^{-1} \leqslant c_{2} \inf _{\xi \in S^{n-1}}|A(t, \nu) \xi|
$$

for some positive constants $c_{1}, c_{2}$, where $\|\cdot\|$ is a norm on the space of matrices. Thus if we prove that

$$
\sup _{1 \leqslant t \leqslant 2}\left\|A(t, \nu)^{-1}\right\| \leqslant c m(\nu)^{-1}
$$

we have that

$$
\inf \left\{|A(t, \nu) \xi|: \xi \in S^{n-1}, 1 \leqslant t \leqslant 2\right\} \geqslant c m(\nu),
$$

which proves Lemma 3.

To prove (3.3), we note that

$$
\operatorname{det} A(t, \nu)=\nu_{1} \cdots \nu_{n} t^{\gamma-n(n+1) / 2} \alpha_{1} \cdots \alpha_{n} \prod_{i>j}\left(\alpha_{i}-\alpha_{j}\right) .
$$

Let adj $A(t, \nu)$ be the adjugate matrix of $A(t, \nu)$. Then it is easy to see that

$$
\sup _{1 \leqslant t \leqslant 2}\|\operatorname{adj} A(t, \nu)\| \leqslant c\left|\nu_{1}\right|\left|\nu_{2}\right| \cdots\left|\nu_{n}\right| m(\nu)^{-1} .
$$

Since $A(t, \nu)^{-1}=\{\operatorname{det} A(t, v)\}^{-1} \operatorname{adj} A(t, \nu)$, (3.3) follows from (3.4) and (3.5). This completes the proof of Lemma 3. 
Proof of Lemma 2. By Lemmas 3, 4 and 5 we have

$$
\left|n_{\nu}(\xi)\right| \leqslant c\{1+m(\nu)|\xi|\}^{-1 / n}
$$

for all $\nu \in S^{n-1}$ and for all $\xi \in \mathbf{R}^{n}$. Note that

$$
\rho^{\tau}(\xi) \leqslant c|\xi| \text { for }|\xi| \geqslant 1 .
$$

Lemma 2 follows from (3.6) and (3.7). This completes the proof of Lemma 2. Here we prove the Main Lemma.

Proof of Main Lemma. Set

$$
I(z, t, \xi)=\int_{S^{n-1}}\left|\eta\left(\delta_{t} \xi\right) n_{\nu}\left(\delta_{t} \xi\right)+\rho^{z}\left(\delta_{t} \xi\right)\left(1-\eta\left(\delta_{t} \xi\right)\right) n_{\nu}\left(\delta_{t} \xi\right)-\hat{\phi}\left(\delta_{t} \xi\right)\right|^{2} d \nu
$$

Then we have

$$
\int_{\mathbf{R}^{n}} g_{z}^{2}(f)(x) d x=\int_{\mathbf{R}^{n}}|\hat{f}(\xi)|^{2}\left(\int_{0}^{\infty} I(z, t, \xi) \frac{d t}{t}\right) d \xi .
$$

We estimate $I(z, t, \xi)$ for $|\xi|=1$. If $0<t \leqslant 1$ and $|\xi|=1$, then

$$
I(z, t, \xi)=\int_{S^{n-1}}\left|n_{\nu}\left(\delta_{t} \xi\right)-\hat{\phi}\left(\delta_{t} \xi\right)\right|^{2} d \nu \leqslant c t^{2 \tau},
$$

since $\hat{\phi}(0)=n_{\nu}(0)=1$. If $1 \leqslant t \leqslant 100$ and $|\xi|=1$, then

$$
I(z, t, \xi) \leqslant c_{z} \text { for some } c_{z}>0 .
$$

If $t>100$ and $|\xi|=1$, we have

$$
I(z, t, \xi)=\int_{S^{n-1}}\left|\rho^{z}\left(\delta_{t} \xi\right) n_{\nu}\left(\delta_{t} \xi\right)-\hat{\phi}\left(\delta_{t} \xi\right)\right|^{2} d \nu .
$$

Note that

$$
\int_{S^{n-1}}\left\{1+m(\nu) t^{\tau}\right\}^{-2 / u} d \nu \leqslant c t^{-2 \tau / u} \quad \text { if } u>n .
$$

Thus by Lemma 2 we have, for each $u$ with $u>n$, that

$$
I(z, t, \xi) \leqslant c_{z} t^{-2 \tau u^{-1}+2 \operatorname{Re} z} \quad \text { if } t>100 \text { and }|\xi|=1 .
$$

By (3.9), (3.10) and (3.11) we have

$$
\sup _{\xi \in S^{n-1}} \int_{0}^{\infty} I(z, t, \xi) \frac{d t}{t} \leqslant c_{z} \quad \text { for } \operatorname{Re} z<\frac{\tau}{n} .
$$

By (3.8), this proves the Main Lemma.

4. Proof of the theorem. For a locally integrable function $f$ on $\mathbf{R}^{n}$, set

$$
N_{\nu, h} f(x)=\frac{1}{h} \int_{h}^{2 h} f\left(x-\delta_{t} \nu\right) d t \quad\left(\nu \in S^{n-1}, h>0\right)
$$

and

$$
N_{\nu} f(x)=\sup _{\varepsilon>0} \frac{1}{\varepsilon} \int_{0}^{\varepsilon}\left|N_{\nu, h} f(x)\right| d h .
$$


Let

$$
N_{\nu}^{(z)} f(x)=\sup _{\varepsilon>0} \frac{1}{\varepsilon} \int_{0}^{\varepsilon}\left|N_{\nu, t}^{(z)} f(x)\right| d t .
$$

Note that $N_{\nu} f(x)=N_{\nu}^{(0)} f(x)$ if $f \in C_{0}^{\infty}\left(\mathbf{R}^{n}\right)$ (see Stein-Wainger [5, p. 1266]).

In order to prove the theorem we need the following lemmas.

Lemma 6. There is a positive constant $c$ such that

$$
\int_{S^{n-1}}\left\{N_{\nu}^{(z)} f(x)\right\}^{2} d \nu \leqslant c\left\{g_{z}^{2}(f)(x)+\sup _{t>0}\left|\phi_{t} * f(x)\right|^{2}\right\} .
$$

Lemma 7. If $-\gamma<\operatorname{Re} z<-\gamma+\tau$, then

$$
\left[\int_{\mathbf{R}^{n}}\left\{\sup _{\nu \in S^{n-1}} N_{\nu}^{(z)} f(x)\right\}^{p} d x\right]^{1 / p} \leqslant c_{p, z}\|f\|_{p} \quad \text { for } 1<p<\infty .
$$

Lemma 8. For $1<p<\infty$, we have

$$
\left[\int_{\mathbf{R}^{n}}\left\{\int_{S^{n-1}} N_{\nu} f(x) d \nu\right\}^{p} d x\right]^{1 / p} \leqslant c_{p}\|f\|_{p}
$$

The proof of Lemma 6 is straightforward. We omit the proof (see [5, p. 1265]).

Proof of Lemma 7. Recall that

$$
N_{\nu, t}^{(z)} f(x)=U_{t}^{(z, \nu)} * f(x)+L_{t}^{(\nu)} * f(x)
$$

where

$$
\left(U^{(z, \nu)}\right)^{\wedge}(\xi)=\rho^{z}(\xi)(1-\eta(\xi)) n_{\nu}(\xi), \quad\left(L^{(\nu)}\right)^{\wedge}(\xi)=\eta(\xi) n_{\nu}(\xi) .
$$

Since

$$
L^{(\nu)}(x)=\int_{1}^{2} \check{\eta}\left(x-\delta_{t} \nu\right) d t
$$

we have

$$
\sup _{\nu \in S^{n-1}}\left|L^{(\nu)}(x)\right| \leqslant c\{1+\rho(x)\}^{-\gamma-1} .
$$

If $-\gamma<\operatorname{Re} z<0$, then

$$
U^{(z, \nu)}(x)=\int_{1}^{2} v^{(z)}\left(x-\delta_{t} \nu\right) d t,
$$

where $v^{(z)}$ satisfies the inequalities:

$$
\begin{array}{ll}
\left|v^{(z)}(x)\right| \leqslant c_{z}\{\rho(x)\}^{-\gamma-1} & \text { if }|x| \geqslant 1, \\
\left|v^{(z)}(x)\right| \leqslant c_{z}\{\rho(x)\}^{-\gamma-\operatorname{Re} z} & \text { if }|x| \leqslant 1
\end{array}
$$

(see Stein-Wainger [5, p. 1279]). 
Thus if $-\gamma<\operatorname{Re} z<-\gamma+\tau$, then

$$
\sup _{\nu \in S^{n-1}}\left|U^{(z, \nu)}(x)\right| \leqslant c_{z}\{1+\rho(x)\}^{-\gamma-1} .
$$

By (4.1), (4.2) and (4.3), using Lemma 1, we obtain

$$
\left\|\sup _{\nu \in S^{n-1}} \sup _{t>0}\left|N_{\nu, t}^{(z)} f\right|\right\|_{p} \leqslant c_{p, z}\|f\|_{p} \quad \text { for } 1<p \leqslant \infty .
$$

This completes the proof of Lemma 7.

In order to prove Lemma 8, we need the following lemmas.

LEMMA 9. If $-1<\operatorname{Re} z<\tau / n$, then

$$
\left\|N_{\nu}^{(z)} f\right\|_{2} \leqslant c_{z} m(\nu)^{-1 / n}\|f\|_{2} .
$$

LEMMA 10. There is $\sigma_{2}>0$ such that if $0<\sigma_{3}<\sigma_{2}$ and $-\sigma_{2}<\operatorname{Re} z<-\sigma_{3}$, then

$$
\left\|N_{\nu}^{(z)} f\right\|_{p} \leqslant c_{p, z}\|f\|_{p} \quad \text { for } 1<p \leqslant \infty
$$

where $c_{p, z}$ is independent of $\nu$.

Arguing as in the proof of Lemma 5-4 of Stein-Wainger [5], and using Lemma 2, we obtain Lemma 9.

Reading the proof of Lemma 5-5 of Stein-Wainger [5] carefully, we obtain Lemma 10.

Proof of Lemma 8. By Lemma 9 and Lemma 10, using the analytic interpolation theorem as in $[5, \S 5]$, we have

$$
\left\|N_{\nu} f\right\|_{p} \leqslant c_{p} m(\nu)^{-1 / n}\|f\|_{p}
$$

for $1<p<\infty$. Lemma 8 follows from Minkowski's inequality for integrals and (4.4). This completes the proof of Lemma 8.

Now we prove the theorem.

Proof of The Theorem. By Lemma 1, the Main Lemma and Lemma 6 we have

$$
\left[\int_{\mathbf{R}^{n}} \int_{S^{n-1}}\left\{N_{\nu}^{(z)} f(x)\right\}^{2} d \nu d x\right]^{1 / 2} \leqslant c_{z}\|f\|_{2}
$$

for $\sigma_{1} / 2<\operatorname{Re} z<\sigma_{1}$.

By (4.5) and Lemma 7, using the analytic interpolation theorem with respect to the mixed norms, we have

$$
\left[\int_{\mathbf{R}^{n}}\left\{\int_{S^{n-1}}\left(N_{\nu} f(x)\right)^{s} d \nu\right\}^{p / s} d x\right]^{1 / p} \leqslant c\|f\|_{p}
$$

if

$$
s<\frac{2\left(\sigma_{1}+\gamma-\tau\right)}{\gamma-\tau}, \quad \frac{2\left(\sigma_{1}+\gamma-\tau\right)}{2 \sigma_{1}+\gamma-\tau}<p<\frac{2\left(\sigma_{1}+\gamma-\tau\right)}{\gamma-\tau}
$$


By (4.6) and Lemma 8, via an interpolation theorem, we obtain

$$
\left[\int_{\mathbf{R}^{n}}\left\{\int_{S^{n-1}}\left(N_{\nu} f(x)\right)^{2} d \nu\right\}^{p / 2} d x\right]^{1 / p} \leqslant c_{p}\|f\|_{p}
$$

for

$$
p>\frac{2\left(2 \sigma_{1}+\gamma-\tau\right)}{4 \sigma_{1}+\gamma-\tau}
$$

Note that if $f \geqslant 0$, then

$$
M_{\nu} f(x) \leqslant c N_{\nu} f(x) \quad(\text { see }[5, \text { p. 1265]). }
$$

By (4.7) and (4.8) we obtain the theorem. This completes the proof of the theorem.

REMARK. We omit the technical details in the interpolation argument given in the proof of the theorem since they are similar to those of Stein-Wainger [5, §5].

\section{Application. Let}

$$
K_{\varepsilon}(x, y)=\varepsilon^{-\gamma} N(x, x-y) \chi_{[0,1]}(\rho(x-y) / \varepsilon) \quad(\varepsilon>0),
$$

where $\chi_{[0,1]}$ is the characteristic function of the interval $[0,1]$, and $N$ satisfies the conditions:

$$
\begin{gathered}
N\left(x, \delta_{t} y\right)=N(x, y) \quad \text { for all } t>0, \\
\sup _{x \in \mathbf{R}^{n}} \int_{S^{n-1}}|N(x, \nu)|^{2} d \nu<\infty
\end{gathered}
$$

Set

$$
T_{\varepsilon}(f)(x)=\int_{\mathbf{R}^{n}} f(y) K_{\varepsilon}(x, y) d y, \quad T^{*}(f)(x)=\sup _{\varepsilon>0}\left|T_{\varepsilon}(f)(x)\right| .
$$

As an application of the theorem we obtain the following result.

COROllary. If

$$
p>\frac{2\{n \gamma-(n-2) \tau\}}{n \gamma-(n-4) \tau}
$$

then

$$
\left\|T^{*}(f)\right\|_{p} \leqslant c_{p}\|f\|_{p}
$$

Proof. Arguing as in Calderón-Zygmund [1], we have $T^{*}(f)(x) \leqslant c M(f)(x)$. Thus the corollary is an immediate consequence of the theorem. This completes the proof.

\section{REFERENCES}

1. A. P. Calderón and A. Zygmund, On singular integrals, Amer. J. Math. 78 (1956), 289-309.

2. R. Fefferman, On an operator arising in the Calderon-Zygmund method of rotations and the Bramble-Hilbert lemma, Proc. Nat. Acad. Sci. U.S.A. 80 (1983), 3877-3878.

3. N. M. Rivière, Singular integrals and multiplier operators, Ark. Mat. 9 (1971), 243-278.

4. E. M. Stein and S. Wainger, The estimation of an integral arising in multiplier transformations, Studia Math. 35 (1970), 101-104.

5. $1239-1295$. , Problems in harmonic analysis related to curvature, Bull. Amer. Math. Soc. 84 (1978), 